\title{
Nonlinear dynamics and global analysis of a heterogeneous Cournot duopoly with a Local Monopolistic Approach versus a Gradient Rule with endogenous reactivity
}

\author{
Fausto Cavalli ${ }^{\mathrm{a}, *}$, Ahmad Naimzada ${ }^{\mathrm{a}}$, Fabio Tramontana ${ }^{\mathrm{b}}$ \\ ${ }^{a}$ Department of Economics, Management and Statistics, University of Milano-Bicocca, \\ Milan, Italy \\ ${ }^{b}$ Department of Economics and Management University of Pavia, Italy
}

\begin{abstract}
We study a heterogeneous duopolistic Cournotian game, in which the firms, producing a homogeneous good, have reduced rationality and respectively adopt a "Local Monopolistic Approximation" (LMA) and a gradient-based approach with endogenous reactivity, in an economy characterized by isoelastic demand function and linear total costs. We give conditions on reactivity and marginal costs under which the solution converges to the Cournot-Nash equilibrium. Moreover, we compare the stability regions of the proposed oligopoly to a similar one, in which the LMA firm is replaced by a best response firm, which is more rational than the LMA firm. We show that, depending on costs ratio, the equilibrium can lose its stability in two different ways, through both a flip and a Neimark-Sacker bifurcation. We show that the nonlinear, noninvertible map describing the model can give rise to several coexisting stable attractors (multistability). We analytically investigate the shape of the basins of attractions, in particular proving the existence of regions known in the literature as lobes.
\end{abstract}

\section{Introduction}

Starting from the seminal book of Cournot in 1838 [1], the oligopolistic market structures have been deeply studied and analyzed as a significant subject of economic dynamics and game theory. In an oligopolistic framework, the market is supplied by only a few firms, two in the particular case of a duopoly, and each firm chooses its strategy taking into account at the same time both its own actions and those of their competitors.

\footnotetext{
* Corresponding author

Email addresses: fausto.cavalli@unimib.it (Fausto Cavalli), ahmad.naimzada@unimib.it (Ahmad Naimzada), fabio.tramontana@unipv.it (Fabio Tramontana)
} 
Assuming that each firm in endowed with an high degree of rationality and with cognitive and computational skills that allow them to exactly know the demand curve of the produced good and their technology (represented by the cost function), an oligopoly can be studied in a static game setting by means of the notion of Nash equilibrium. Having each firm perfect foresight of the next period productions, the players are able to solve a one period optimization problem.

Weakening the degree of rationality of the firms, a dynamic adjustment arises and new scenarios appear, as even a simple duopoly can give rise to complex dynamic phenomena, as shown in the works of Rand [2] and Poston and Stewart [3] in 1978. The dynamic behavior of oligopolistic models have been intensively investigated: among all the contributions, we can mention the key work of Puu in 1991 [4], which proposed a duopoly based on unimodal reaction functions derived solving an optimization problem for profit functions. Studying the case of constant marginal costs and, starting from Cobb-Douglas type preferences for the consumer, of isoelastic demand function, Puu showed that each competitor outputs can evolve through a period doubling sequence of flip bifurcations which leads finally to chaos.

The work of Puu started a wide literature about duopolistic and oligopolistic market and lead off different research strands. One of these, deeply investigated in the last years, concerns the analysis of decisional mechanism, dissimilar to the best response rule, based on different (usually lower) degrees of informational and computational abilities. Among these mechanisms, we can mention the gradient-like mechanism and "Local Monopolistic Approximation" (LMA).

The LMA is an approach which was proposed for the first time by Silvestre in [5] with the name of "Strong Monopolistic Equilibrium", and then reconsidered by Tuinstra [6], Bischi et al. [7], Naimzada and Sbragia [8] and applied in a monopolistic setting by Naimzada and Ricchiuti [9]. In the LMA, the oligopolists do not know the market demand function, and conjecturing it is linear, they proceed estimating such a linear function through the knowledge of the local true slope of the demand curve and the knowledge of the current market state in terms of quantities and price. We remark that Silvestre in [5] already introduced such concept, he

In the gradient-like mechanism, the players, which are not requested to have a complete knowledge of the demand and cost function, do not choose their strategy solving any optimization problem, but they use a local estimate of the marginal profit. The output level is updated by following the direction of an increase of the corresponding profit function, which is indeed regulated by its gradient. The reactivity of this adjustment is governed by a parameter, which can be set in different ways. In particular, it can be constant with respect to the produced quantity or endogenously dependent from it, to enforce its dependence on the firm size. The constant reactivity gradient mechanism is discussed in the book of Varian [10] and by Corchon and Mas-Colell in [11], while duopolies involving a firm following it were studied by Angelini et al. [12] and Cavalli et al. [13]. Conversely, the endogenous reaction was studied by Bischi and Naimzada [14], Bischi et al. [15, 16], Agiza et al. [17], Tramontana [18] and 
more recently by Askar in $[19,20]$. In the present work, we consider endogenous reaction.

This paper belongs to the literature about heterogeneous oligopolies, in the sense that the firms adopt different decisional mechanisms. The heterogeneous framework was studied for example in the articles by Leonard and Nishimura [21], Den-Haan [22], Agiza and Elsadany [23, 24], Angelini et al. [12], Tramontana [18], Dubiel-Teleszynski [25], Anufriev et al. [26]. These works concern the coupling of a best response decisional mechanism with the gradient like decisional mechanism. In our work, we want to consider a duopoly made up by two firms which adopt the gradient-like and the Local Monopolistic Approximations. This work belongs to the research strand in which we are investigating several aspects of heterogeneous duopolies. In particular, we focus on the effect of different degrees of rationality in Cavalli and Naimzada [27], where we compared decisional mechanisms based on best response adjustments involving different degrees of rationality. Conversely, in Cavalli al. [13] we study the effect of exogenous reactivity.

We remark that the economic structure in which we study our model is similar to those proposed by Angelini et al. [12] and Tramontana [18] with respect to the isoelastic demand function and to the constant marginal costs. In particular, the duopoly we consider differs from that studied in [18] only for the second firm decisional mechanism, since in [18] the second firm chooses its output using the classical best response with static expectations, so the two duopolies are directly comparable.

The main results of this work concern the study of the local stability of the equilibrium; the investigation of the possible routes of destabilization for the equilibrium point; the study of global properties of the two dimensional map that models the oligopoly.

Concerning the first result, after obtaining the constraints on the costs ratio to have a stable equilibrium, we compare the resulting stability region of the Nash equilibrium with that of the duopoly studied in [18]. We prove that, despite of the reduced rationality of one of the firms, the duopoly studied in this work can present a larger stability region. In particular, the stability region is larger for the duopoly studied in the present work when the costs are sufficiently different, while it is larger for the gradient versus best response duopoly when the costs are more comparable.

Concerning the second result, we show that the equilibrium point can be destabilized by means of both a flip and a Neimark-Sacker bifurcation, depending on the costs ratio. In particular, it is shown that, when the cost ratio is sufficiently favorable to the firm which adopts the gradient-like decisional mechanism, the scenario presents a Neimark-Sacker bifurcation, while the flip bifurcation occurs when the costs structure is favorable to the firm using linear approximation. This result improves the knowledges about dynamics involving gradient rule, and further confirms recent studies showing that both NeimarkSacker and flip bifurcations appear to be the possible destabilization, as shown in $[15,16,12,18,13])$, especially when a gradient-rule dynamic is present.

Concerning the third result, we show that dealing with a nonlinear, and in 
particular noninvertible map, the only local stability analysis does not permit to fully understand the dynamics. Several stable attractors may coexist (multistability) giving rise to a situation of path dependency. The shape of the basins of attractions can be quite complicated, especially because we deal with a twodimensional map characterized by a denominator that can vanish along a line, and one component of the map takes the form $0 / 0$ at one point. In these cases the basins of attraction can be characterized by particular shapes known in the literature as lobes.

The paper is organized as follows. In Section 2, we introduce the model and the nonlinear system describing the dynamics of the productions of the firms. In Section 3, we determine the conditions under which the Nash equilibrium is locally stable. In Section 4, we investigate the global behavior of the system. In Section 5 we conclude and we propose some future developments. In Appendix we collect the proof of the propositions.

\section{Model}

The economic setting in which we study the duopolistic market consists of isoelastic demand function and constant marginal costs. In particular, if we suppose that the economy is populated by $n$ agents, each having Cobb-Douglas preferences, we can express the utility function of the representative $j$ th agent by $U_{j}\left(q_{j}\right)=\prod_{k=1}^{m}\left(q_{j}^{k}\right)^{\alpha_{j}^{k}}$, where $q_{j}=\left(q_{j}^{1}, q_{j}^{2}, \ldots, q_{j}^{m}\right)$ is the vector of the quantities of the $m$ goods. If we introduce a budget constraint $\sum_{k=1}^{m} p_{k} q_{j}^{k} \leq y^{j}$ for the $j$ th agent, being $p_{k}$ the price of commodity $k$ and $y^{j}$ is the income of the $j$ th agent, we can solve the constrained maximization problem obtaining the demand function

$$
q_{j}^{k}=\frac{\alpha_{j}^{k} y^{j}}{p_{k}} .
$$

Focusing only on one market, suppressing the index $k$, and summing over all the agents, we have the aggregated demand, from which, after setting $\sum_{j} \alpha_{j} y^{j}=1$, we have the constant elasticity inverse demand function

$$
p(Q)=\frac{1}{Q}
$$

where $Q=\sum_{j} q_{j}$.

In the present, work we assume that the industry is composed by two firms (indexed by $i=1,2$ ), which produce perfect substitute goods $q_{1}$ and $q_{2}$. If $c_{i}>0$ represent the (constant) marginal costs of each duopolist, we have that the linear cost functions are expressed by

$$
C_{i}\left(q_{i}\right)=c_{i} q_{i} \quad i=1,2 .
$$

In this framework, the profit of the firms are

$$
\Pi_{1}\left(q_{1}, q_{2}\right)=\frac{q_{1}}{Q}-c_{1} q_{1}, \quad \Pi_{2}\left(q_{1}, q_{2}\right)=\frac{q_{2}}{Q}-c_{2} q_{2}
$$


respectively. This situation can be analyzed in a game theoretic context, in which the players are the two duopolist, the strategies are given by the set of all the productions $\left(q_{i} \geq 0\right)$ and the payoff functions are the profit functions $(2)$. It is easy to see that, as in Puu [4], we have one Nash equilibrium given by

$$
E=\left(q_{1}^{N}, q_{2}^{N}\right)=\left(\frac{c_{2}}{\left(c_{1}+c_{2}\right)^{2}}, \frac{c_{1}}{\left(c_{1}+c_{2}\right)^{2}}\right) .
$$

It is worth noticing that the Nash equilibrium notion can be very demanding in terms of rationality and information set possessed by the players. In this work, we consider two different mechanisms which imply a reduced degree of rationality, the "Local Monopolistic Approximation" (LMA) and the gradient adjustment mechanism. The description of these two mechanism can be found in [13]. For the sake of completeness, we briefly summarize them.

Under the gradient adjustment mechanism, which we assume that is adopted by the first boundedly rational firm, the firm increases or decreases its output according to the information given by the last period profit variation. Such variation is represented by the derivative of the profit function with respect to its strategy $q_{1}$, namely the marginal profit $\phi_{1}\left(q_{1}, q_{2}\right)=\partial_{q_{1}} \Pi_{1}\left(q_{1}, q_{2}\right)$

$$
\phi_{1}\left(q_{1}, q_{2}\right)=\frac{q_{2}}{\left(q_{1}+q_{2}\right)^{2}}-c_{1}
$$

If we assume that the relative variation in production quantities is proportional to the marginal profits,

$$
\frac{q_{1, t+1}-q_{1, t}}{q_{1, t}}=\alpha \phi_{1}\left(q_{1, t}, q_{2, t}\right)
$$

where $\alpha>0$ represents the speed of adjustment, the resulting dynamic is given by

$$
q_{1, t+1}=q_{1, t}+\alpha q_{1, t}\left(\frac{q_{2, t}}{\left(q_{1, t}+q_{2, t}\right)^{2}}-c_{1}\right) .
$$

We remark that trajectories of (4) may become negative. In what follows, we only focus on such initial data and parameters for which $q_{1, t}$ stays positive for $t>0$. Otherwise, the maximum between $q_{1, t+1}$ and 0 should be taken.

The "Local Monopolistic Approximation", which is assumed to be the decisional mechanism of the second firm, is a bounded rational adjustment process in which the firm possesses only a limited knowledge of the demand function. It was studied in $[7,28]$, and it is founded on a best response mechanism with respect to a linear approximation of the true demand function. Suppose that the firm knows at time $t$ the market price $p\left(Q_{t}\right)$ and the corresponding produced quantity $Q_{t}$. By means of market research, studying market values in a neighborhood of $\left(p\left(Q_{t}\right), Q_{t}\right)$, the firm can correctly compute the slope of the price function

$$
\partial_{q_{1, t}} f\left(q_{1, t}, q_{2, t}\right)=f^{\prime}\left(Q_{t}\right)
$$


estimating, for example, the effects of small quantity or price variations, as detailed in $[7,28]$. Then, using (5) and the price $p_{t}$, the firm conjectures a linear price function

$$
p^{e}\left(Q^{e}\right)=p\left(Q_{t}\right)+p^{\prime}\left(Q^{e}\right)\left(Q_{t+1}^{e}-Q_{t}\right),
$$

where we set $Q^{e}=q_{1, t+1}^{e}+q_{2, t+1}$. With $q_{1, t+1}^{e}$ we indicate the output that the second oligopolists expects that its opponent $i=1$ produces at time $t+1$. If we consider static expectations $\left(q_{1, t+1}^{e}=q_{1, t}\right)$, we can rewrite (6) as

$$
p^{e}\left(Q^{e}\right)=\frac{1}{q_{1, t}+q_{2, t}}-\frac{1}{\left(q_{1, t}+q_{2, t}\right)^{2}}\left(q_{2, t+1}-q_{2, t}\right),
$$

where we used (1). Maximizing the expected profit (2) at time $t+1$

$$
q_{2, t+1}=\arg \max _{q_{2, t+1}}\left[p^{e}\left(Q^{e}\right) q_{2, t+1}-c_{2} q_{2, t+1}\right],
$$

we can obtain the output assumed for time $t+1$. To this end, we notice that

$p^{e}\left(Q^{e}\right) q_{2, t+1}-c_{2} q_{2, t+1}=-\frac{q_{2, t+1}^{2}}{\left(q_{1, t}+q_{2, t}\right)^{2}}+q_{2, t+1}\left(\frac{1}{q_{1, t}+q_{2, t}}+\frac{q_{2, t}}{\left(q_{1, t}+q_{2, t}\right)^{2}}-c_{2}\right)$,

so that solving $\partial_{q_{2, t+1}}\left(p^{e}\left(Q^{e}\right) q_{2, t+1}-c_{2} q_{2, t+1}\right)=0$ we find

$$
q_{2, t+1}=\frac{1}{2} q_{2, t}+\frac{1}{2}\left(1-c_{2}\left(q_{1, t}+q_{2, t}\right)\right)\left(q_{1, t}+q_{2, t}\right),
$$

which, as it can be easily checked, satisfies second order condition too. We remark that if $q_{1, t} \geq 1 / c_{2}$, then $p^{e} \leq c_{2}$ and the previous maximization problem would provide $q_{2, t+1} \leq 0$ and the production level of the firm would be null. As we only focus on positive trajectories, we avoid to take into account such situation.

The resulting duopoly game is obtained matching the gradient firm (4) and the LMA firm (8), obtaining the following discrete dynamical system

$$
T_{1}\left(q_{1}, q_{2}\right):\left\{\begin{array}{l}
q_{1, t+1}=q_{1, t}+\alpha q_{1, t}\left(\frac{q_{2, t}}{\left(q_{1, t}+q_{2, t}\right)^{2}}-c_{1}\right), \\
q_{2, t+1}=\frac{1}{2} q_{2, t}+\frac{1}{2}\left(1-c_{2}\left(q_{1, t}+q_{2, t}\right)\right)\left(q_{1, t}+q_{2, t}\right) .
\end{array}\right.
$$

We remark that the main difference between the present model (9) and that studied in [13] lies in the gradient adjustment mechanism, which in [13] is considered with exogenous reaction. When the reactivity depends on the output level, it allows taking into account the size of the firm. In fact, larger firms can increase their production more rapidly, as they can make larger investments when the profits increase, as well as in the presence of a negative profit signal, they need to reduce their production in a short time, to avoid bankruptcy risks. 


\section{Local analysis}

In this section we analyze the local properties of the steady states of system (9), whose relation with the Nash equilibrium (3) is made precise in the following proposition.

Proposition 1. The Nash equilibrium (3) is the unique steady state of system (9). $(0,0)$.

The proof is straightforward, also noticing that $(9)$ is not defined for $\left(q_{1}, q_{2}\right)=$

The local stability of the Nash equilibrium is studied in the next proposition, where we make use of

$$
\alpha_{f}=\frac{8 c_{1}+4 c_{2}}{c_{2}\left(7 c_{1}-c_{2}\right)},
$$

and

$$
\alpha_{n s}=\frac{2}{c_{2}-3 c_{1}} .
$$

Proposition 2. Equilibrium (3) is stable provided that

$$
\begin{aligned}
& 0<\frac{c_{1}}{c_{2}} \leq \frac{1}{4} \text { and } \alpha<\alpha_{n s}, \\
& \frac{c_{1}}{c_{2}}>\frac{1}{4} \text { and } \alpha<\alpha_{f} .
\end{aligned}
$$

The equilibrium can become unstable by means of either a flip or NeimarkSacker bifurcation. In fact when $c_{1} / c_{2}>1 / 4$, for $\alpha=\alpha_{f}$, the fixed point $E$ of system (9) becomes unstable by means of a flip bifurcation. Actually, the scenario is more complex, as trajectories can behave differently depending on the initial data. In Figure 1, we report two bifurcation diagrams both corresponding to $c_{1}=0.9, c_{2}=1.62$, but obtained with $\left(q_{1}(0), q_{2}(0)\right)=(0.28,0.11)$ (the red one) and $\left(q_{1}(0), q_{2}(0)\right)=(0.1,0.9)$ (the black one), on varying $\alpha$. We can see that, even before the bifurcation threshold $\alpha_{f} \approx 1.8044$, we can have a period-2 cycle (black diagram) which coexists with the fixed point (red diagram). This suggests the coexistence of different attractors. This will be investigated further in Section 4.

In Figure 2 we report the attractors for $\left(q_{1}(0), q_{2}(0)\right)=(0.28,0.11)$ and several choices of $\alpha$. The dynamic starts with a period-2 cycle, which evolves in period- 4 and period- 8 cycles. Then, chaotic unconnected areas appear, which finally evolve in a chaotic attractor.

Conversely, when $c_{1} / c_{2}<1 / 4$, a Neimark-Sacker bifurcation takes place for $\alpha=\alpha_{n s}$, as shown in Figure 3 for $c_{1}=1$ and $c_{2}=8$, with $\left(q_{1}(0), q_{2}(0)\right)=$ $(0.0889,0.0111)$, as $\alpha$ increases. Above the critical value $\alpha_{n s}$ a Neimark-Sacker bifurcation starts, and lasts until $\alpha \approx 0.48552$, when the quasi-periodic motion is replaced by a periodic one, which then undergoes a sequence of flip bifurcations leading to chaos. In Figure 4, we report some phase plane diagrams, which show the initial quasi-periodic dynamic with attracting invariant closed 


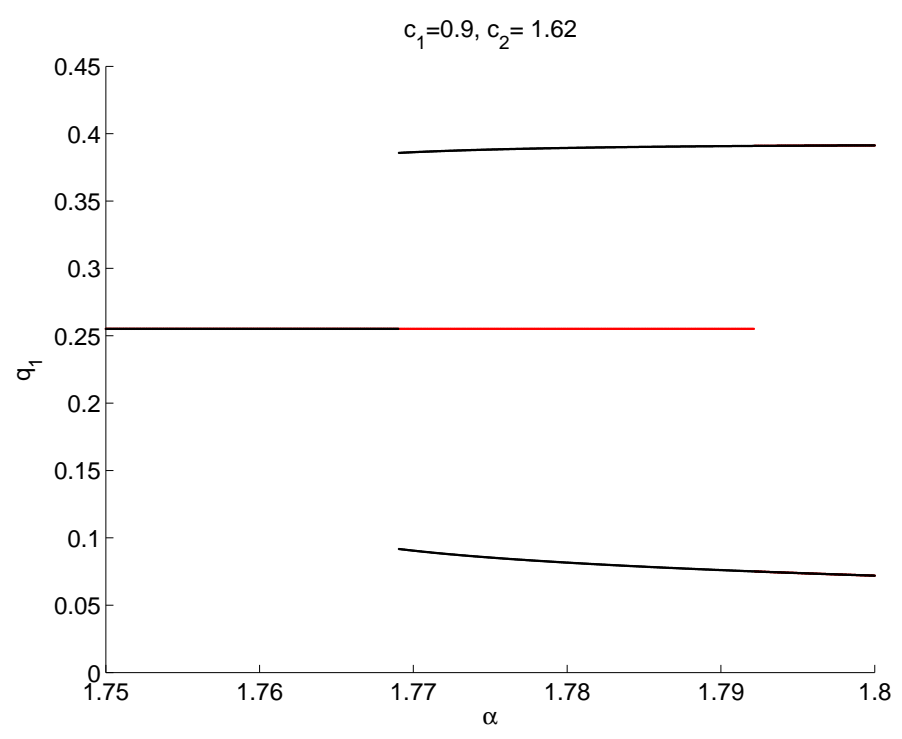

Figure 1: Bifurcation diagram for $c_{1}=0.9, c_{2}=1.62$ and $\alpha \in[1.75,1.8]$ for $\left(q_{1}(0), q_{2}(0)\right)=$ $(0.28,0.11)$ (red) and $\left(q_{1}(0), q_{2}(0)\right)=(0.1,0.9)$ (black). The black trajectory converges for $\alpha>1.77$ to a two point attractor, while the red trajectory converges to the equilibrium.

curves, a successive period-12 cycle and the following 12 unconnected cyclical areas. Increasing further $\alpha$, the previous regions start gathering into four chaotic areas, which finally connect into a quadrilateral shaped area, which becomes increasingly chaotic.

The previous considerations and simulations remark the destabilizing role of the reactivity parameter $\alpha$, which means that output levels of reactive firms follow unstable evolutions.

For ratios $c_{1} / c_{2}$ belonging to $(1 / 7,1 / 3)$, the scenarios are similar to those of the neighboring regions.

We remark that the previous considerations can be read in terms of the way each firm uses to decide its next time output. First of all we notice that when the marginal cost ratio is relatively small $\left(c_{1} / c_{2}<1 / 4\right)$, i.e. when the firm that adopts the gradient mechanism is relatively more efficient, we have a quasi-periodic motion, at least for $\alpha \in\left(\alpha_{n s}, \tilde{\alpha}\right)$ (for example, in the simulation of Figure 3 we have $\tilde{\alpha} \approx 0.48552)$. Such dynamic results in a macro predictable behavior of the output level. Conversely, when the cost ratio is relatively large $\left(c_{1} / c_{2}>1 / 4\right)$, i.e. when the firm that adopts the linear reconstruction mechanism is relatively more efficient, or when $c_{1} / c_{2} \in(0,1 / 4)$ and $\alpha$ is much larger than $\alpha_{n s}$, the dynamic shows first oscillations between two or more different output levels, then it becomes chaotic.

Model (9) is similar to the model studied in [18], in which the second firm uses a naive best reply strategy to maximize its profit. In this case, the resulting 

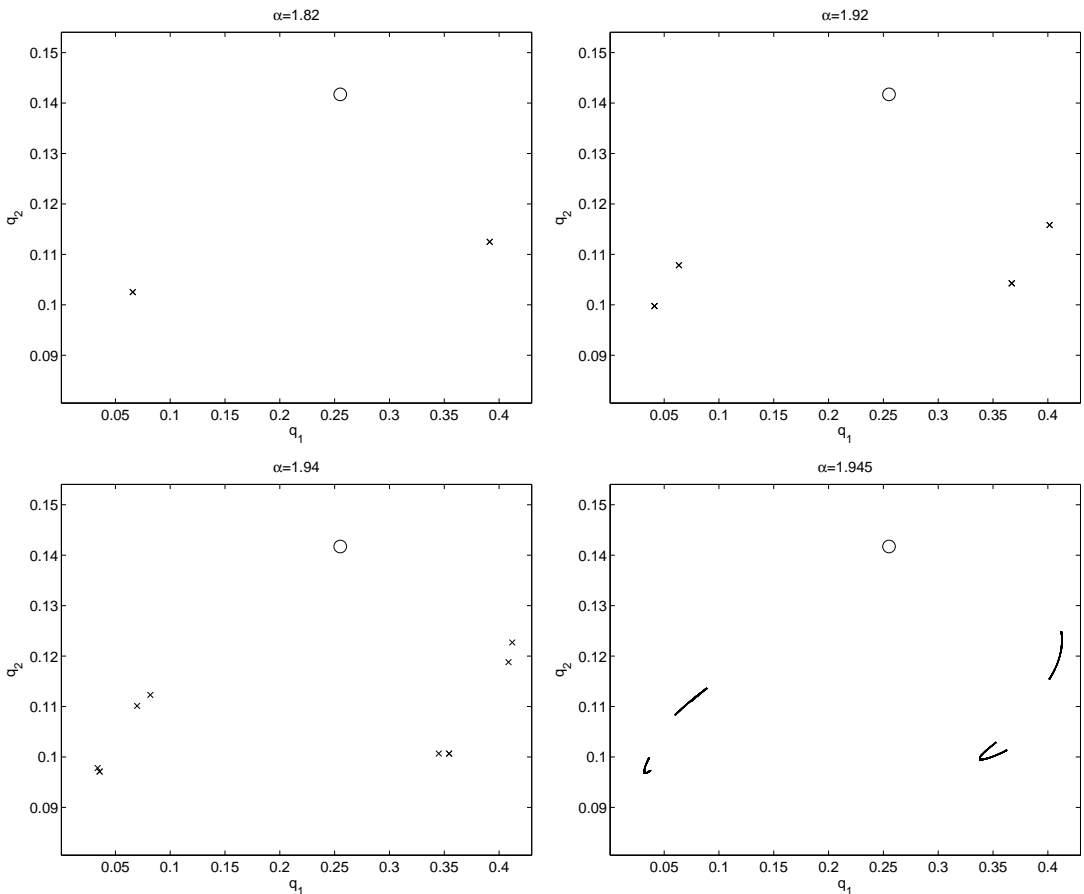

$\alpha=1.95$
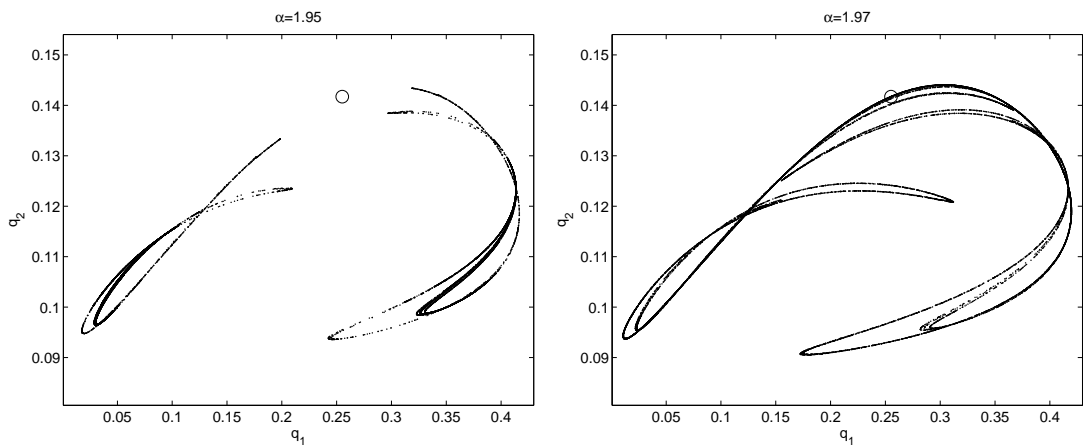

Figure 2: From left to right, top to bottom. Phase plane diagrams for several values of parameter $\alpha$ and marginal costs such that $c_{1} / c_{2}>1 / 3$. The Nash equilibrium is represented by the circle. 


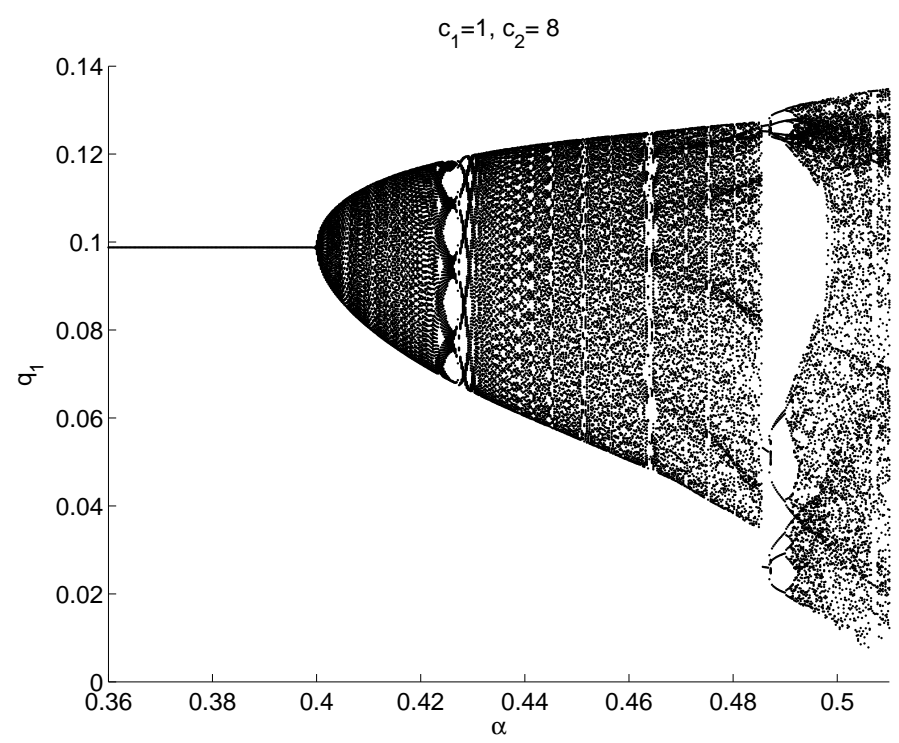

Figure 3: Bifurcation diagram for $c_{1}=1$ and $c_{2}=8$ and $\alpha \in[0.36,0.51]$.

discrete dynamical system is

$$
T_{2}\left(q_{1}, q_{2}\right):\left\{\begin{array}{l}
q_{1, t+1}=q_{1, t}+\alpha q_{1, t}\left(\frac{q_{2, t}}{\left(q_{1, t}+q_{2, t}\right)^{2}}-c_{1}\right), \\
q_{2, t+1}=\sqrt{\frac{q_{1, t}}{c_{2}}}-q_{1, t} .
\end{array}\right.
$$

We want to compare the two models, in particular with respect to their stability region. We underline that the main difference between (9) and (13) lies in the degree of rationality and information implemented by the second firm. As proved in [18], model (13) is stable provided that

$$
\begin{aligned}
& 0<\frac{c_{1}}{c_{2}} \leq \frac{1}{3} \cup \frac{c_{1}}{c_{2}} \geq 3 \text { and } \alpha<\alpha_{n s}^{T}, \\
& \frac{1}{3}<\frac{c_{1}}{c_{2}}<3 \text { and } \alpha<\alpha_{f}^{T},
\end{aligned}
$$

where

$$
\alpha_{f}^{T}=\frac{4\left(c_{1}+c_{2}\right)}{4 c_{1} c_{2}-\left(c_{2}-c_{1}\right)^{2}}
$$

and

$$
\alpha_{n s}^{T}=\frac{2\left(c_{1}+c_{2}\right)}{\left(c_{2}-c_{1}\right)^{2}} .
$$

The comparison between the stability regions of (9) and (13) is investigated in the following theorem. 

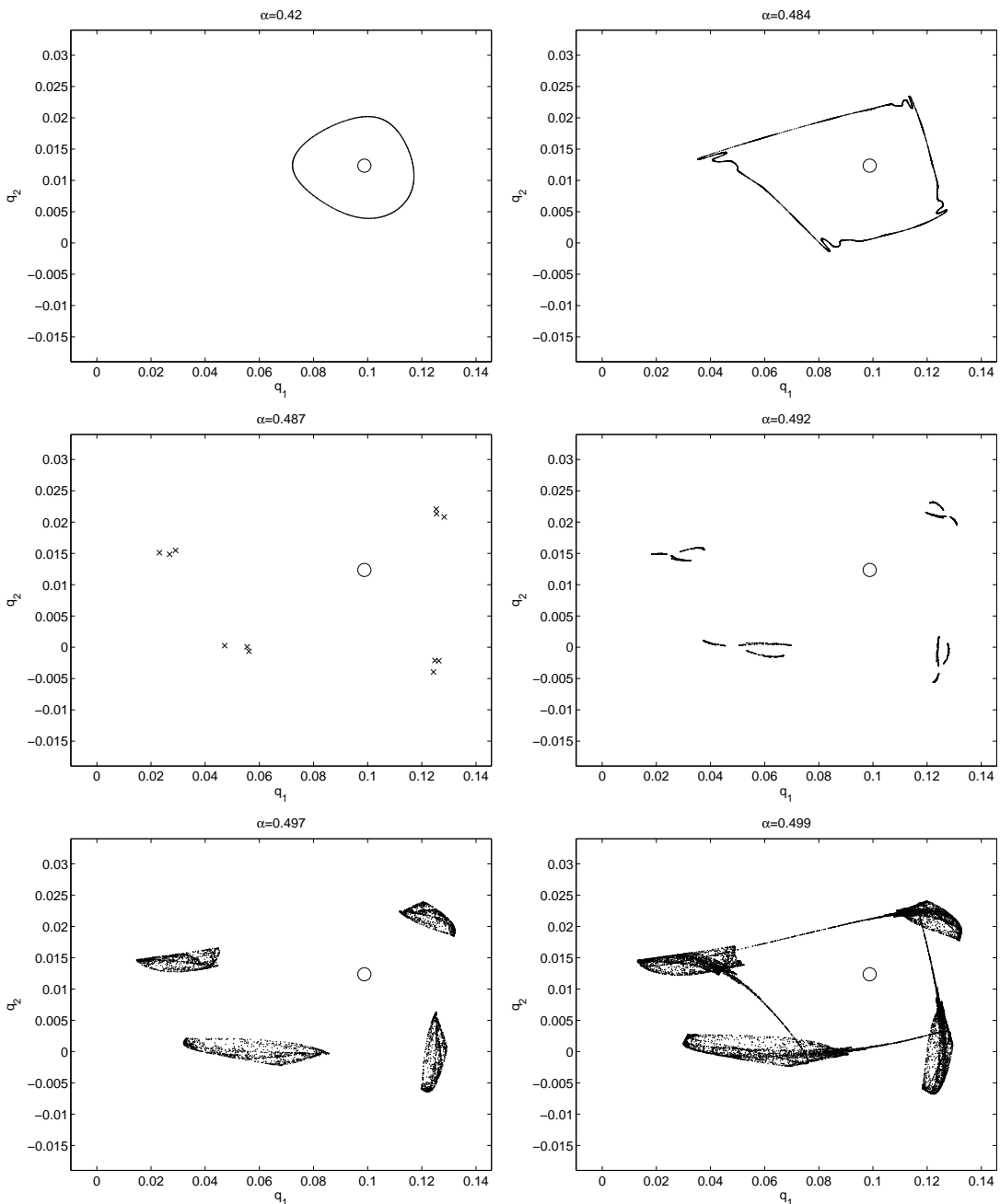

Figure 4: From left to right, top to bottom. Phase plane diagrams for several values of parameter $\alpha$ and marginal costs such that $c_{1} / c_{2}<1 / 7$. The Nash equilibrium is represented by the circle. 

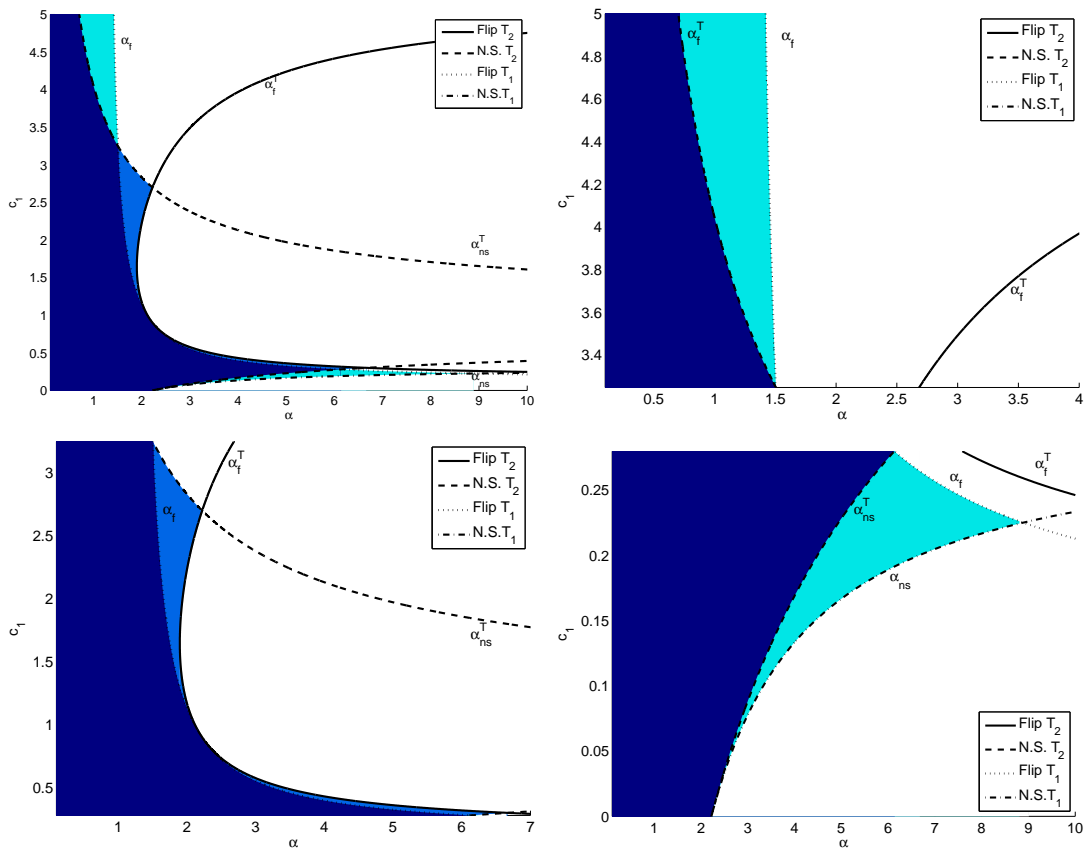

Figure 5: Comparison of the stability regions in space $\left(\alpha, c_{1}\right)$ for model (9) described by map $T_{1}$ and for model (13) described by map $T_{2}$. The curves represent the values (10),(11),(15),(16) above which the bifurcations take place. In particular, the solid and the dashed lines respectively represent the flip and the Neimark-Sacker bifurcation curves for map $T_{2}$, while the dotted and the dash-dotted lines respectively represent the flip and the Neimark-Sacker bifurcation curves for map $T_{1}$. The dark color is used for the region in which both models are stable. The intermediate color is used for the region in which the equilibrium is stable for model (13) and unstable for model (9) is unstable. The light color is used for the region in which the opposite situation occurs. White color represents region in which both models are unstable.

Proposition 3. The stability region of model (9) is larger than the stability region of model (13) for $c_{1} / c_{2}<s_{1}$ and $c_{1} / c_{2}>s_{2}$, while it is smaller for $s_{1}<c_{1} / c_{2}<s_{2}$, where $s_{1}=0.3108$ and $s_{2}=3.6081$.

In Figure 5 we compare the stability regions of the two models for the particular choice $c_{2}=0.9$. The plot illustrates the result of Theorem 3. As we can see, the presence of the more rational firm following the best response rule with static expectations does not always guarantee a wider stability region.

\section{Global analysis}

The considerations we have made until now, in particular the comments about the bifurcation diagram reported in Figure 1, suggest that the local analysis alone does not permit understanding what may happen to the trajectories of (9). 


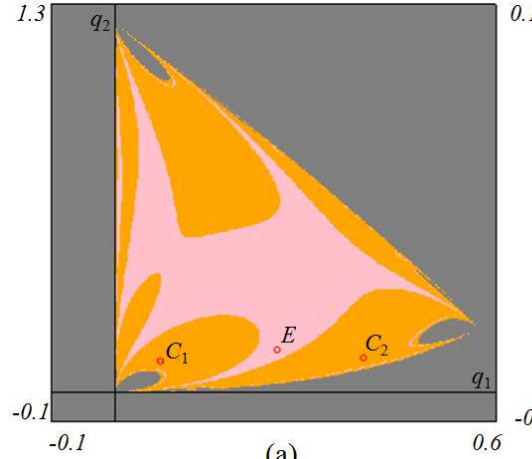

(a)

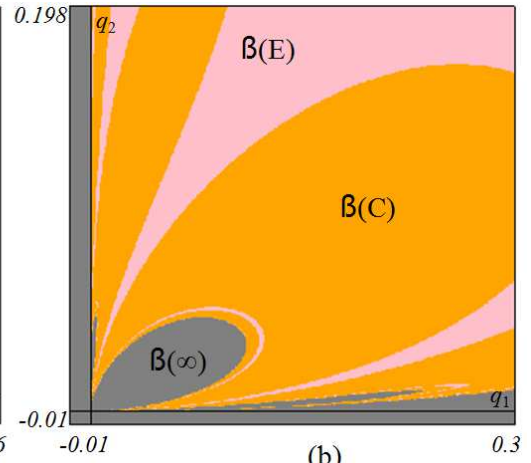

(b)

Figure 6: Phase plane corresponding to $\alpha=1.8, c_{1}=0.9$ and $c_{2}=1.62$. The gray color indicates divergence and unfeasible trajectories, the pink color indicates the basin of attraction of the Nash equilibrium and the orange color denotes the basin of attraction of a cycle of period 2 coexisting with the Nash equilibrium.

In order to see that, let us consider the same set of parameters used in the previous section for the case of $c_{1} / c_{2}>1 / 4\left(c_{1}=0.9\right.$ and $\left.c_{2}=1.62\right)$ and set $\alpha=1.8$. We recall that for these marginal costs the Nash equilibrium can become unstable through a flip bifurcation (see conditions (12)). The threshold value of the speed of adjustment is $\alpha_{f}=1.804$, so the Nash equilibrium is locally stable.

Nevertheless, Figure 6 shows a quite complicated phase plane. First of all, in addition to the pink color characterizing the basin of attraction of the Nash equilibrium and the gray color characterizing divergence (or unfeasible trajectories), there is a third color (orange) denoting the basin of attraction of a cycle of period 2 coexisting with the Nash equilibrium. Multistability is a consequence of the nonlinearity of map $T_{1}$. Moreover, if we want to examine in depth the shape of the basins of attractions to get an insight in the global properties of (9), we must take into consideration two further characteristics of our map: it is non invertible and one of its components has also a denominator vanishing along a one-dimensional subset of the plane, on which a so-called focal point exists.

\subsection{Non invertible maps and Critical curves}

The first element we need to consider is the noninvertibility of $T_{1}$. This means that a point can be characterized by several rank-1 preimages. The rank-1 preimages of a point $P=\left(q_{1}, q_{2}\right)$ are points which are mapped in $P$ in one iteration of the map. Technically speaking, the inverse map $T_{1}^{-1}$ is in general the union of more than one inverse maps of the phase plane. In such cases, the action of the map $T_{1}$ consists in folding and pleating the phase plane, while the inverse map $T_{1}^{-1}$ unfolds it.

Following the notation introduced by Mira et al. in [29] and Abraham et al. in [30], we can subdivide the phase plane into several regions $Z_{i}$, where $i$ is 


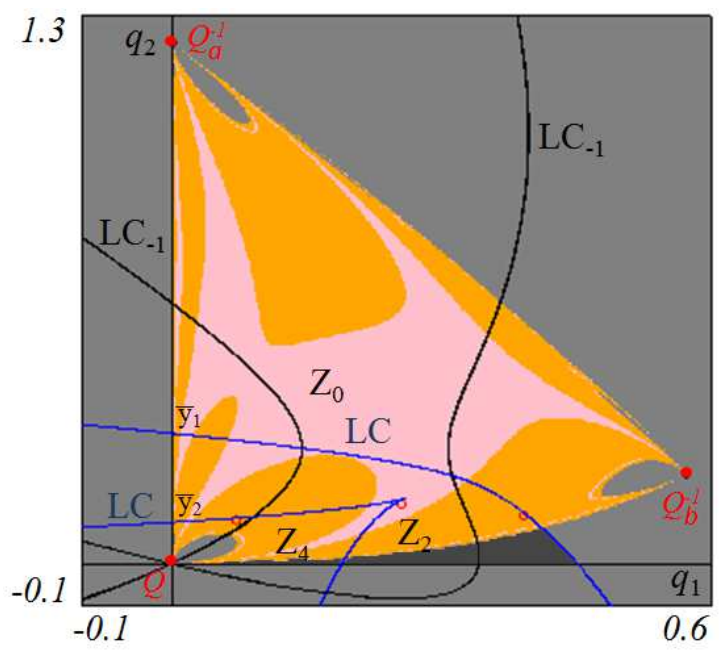

Figure 7: Critical lines for $\alpha=1.8, c_{1}=0.9$ and $c_{2}=1.62$

the number of rank-1 preimages characterizing the points of the corresponding region of the phase plane. The general rule is that two contiguous regions $Z_{i}$ are characterized by a number of preimages that differs by two. This means that, when a point $\left(q_{1}^{\prime}, q_{2}^{\prime}\right)$ of the phase plane crosses a boundary that separates two different regions $Z_{i}$, two real preimages appear or disappear. In particular, the boundary is the locus of points having two (or more) coincident preimages. This locus is a critical curve called $L C$ (from the French Ligne Critique) and the coincident preimages are located on a set called $L C_{-1}$. A critical curve $L C$ generalizes the one-dimensional critical value (local minimum or maximum) to the two-dimensional framework. Similarly, the set $L C_{-1}$ extends the notion of critical point, that is a local extremum point.

Our map (9) does not permit calculating the inverse of each point of the phase plane. Nevertheless, it is still possible to compute the $L C$ curves, by knowing that, for a continuously differentiable map, the set $L C_{-1}$ is included in the set of points where the determinant of the Jacobian matrix $J$ associated to the system (9) vanishes, that is

$$
L C_{-1} \subseteq\left\{\left(q_{1}, q_{2}\right) \in \mathbb{R}^{2} \mid \operatorname{det} J\left(q_{1}, q_{2}\right)=0\right\},
$$

and the critical curves easily come from $L C=T_{1}\left(L C_{-1}\right)$.

For the same parameters used in Figure 6, we report in Figure 7 the numerical computation of the critical lines.

We still don't know which number of rank-1 preimages must be associated to each region $Z_{i}$. Even if we are not able to calculate the number of inverses of each possible point of the phase plane, we can use a subset of it as representative for the other points. In particular, if we calculate the number of preimages of the points of the vertical axis $\left(q_{1}=0\right)$, we can extend the results to all the point belonging to the same region $Z_{i}$. 


\begin{tabular}{|l|l|l|l|}
\hline & 0 & 2 & 4 \\
\hline$\alpha c_{1} \geq 1+\alpha c_{2}$ & & $q_{2}>\bar{y}_{1}$ & $q_{2} \leq \bar{y}_{1}$ \\
\hline $1<\alpha c_{1}<1+\alpha c_{2}$ & $q_{2}>\max \left[\bar{y}_{1}, \bar{y}_{2}\right]$ & $\min \left[\bar{y}_{1}, \bar{y}_{2}\right]<q_{2} \leq \max \left[\bar{y}_{1}, \bar{y}_{2}\right]$ & $q_{2} \leq \min \left[\bar{y}_{1}, \bar{y}_{2}\right]$ \\
\hline$\alpha c_{1}<1$ & $q_{2}<\bar{y}_{1}$ & $q_{2} \geq \bar{y}_{1}$ & \\
\hline
\end{tabular}

Table 1: The rows correspond to the different scenarios according to the values of marginal costs and speed of adjustment. The columns correspond to the number of preimages. The entries are the conditions that must be satisfied to have a certain number of preimages in a certain scenario.

The result is the following

Proposition 4. A point of the vertical axis of the phase plane $\left(0, q_{2}\right)$ can have from 0, 2 or 4 real rank-1 preimages. Setting $\bar{y}_{1}=\frac{1}{2 c_{2}}$ and $\bar{y}_{2}=\frac{\alpha}{8\left[\alpha\left(c_{2}-c_{1}\right)+1\right]}$, if $\alpha c_{1} \geq 1+\alpha c_{2}$, then

- if $\bar{y}_{1} \leq q_{2}$, the point has 4 real preimages;

- if $q_{2}>\bar{y}_{1}$, the point has 2 real preimages.

Instead, if $1<\alpha c_{1}<1+\alpha c_{2}$, then

- if $q_{2} \leq \min \left[\bar{y}_{1}, \bar{y}_{2}\right]$, the point has 4 real preimages;

- if $\min \left[\bar{y}_{1}, \bar{y}_{2}\right]<q_{2} \leq \max \left[\bar{y}_{1}, \bar{y}_{2}\right]$, the point has 2 real preimages;

- otherwise the point has no real preimage.

Finally, if $\alpha c_{1}<1$, then

- if $\bar{y}_{1} \leq q_{2}$, the point has 2 real preimages;

- otherwise the point has no real preimages.

Table 1 further summarizes the results of Proposition 4.

In the case of Figures 6 and 7, we are in the second situation mentioned in the proposition, so we have that the lower portion of the vertical axis (the one containing the origin) is made up by points with 4 real rank- 1 preimages. The points of the middle portion have 2 rank-1 preimages, while the points of the highest portion of the vertical axis have no real preimage. As these points are representative of the whole regions they belong to, we have identified regions $Z_{4}, Z_{2}$ and $Z_{0}$, respectively.

These considerations help to understand the peculiar triangular shape of the basins of attraction drawn in Figures 6 and 7. The origin appears to be a key point, in a sense that will be made precise in the next section. It belongs to the region $Z_{4}$, so it has 4 real preimages. It can be easily proved that two of them are coincident and one is the origin itself, so the origin has only two distinct preimages. They are labeled $Q_{a}^{-1}$ and $Q_{b}^{-1}$. These two points are the other 
vertexes of the pseudo-triangle bounding feasible trajectories. The structure of the basins of attraction around the origin explains also the structure around its preimages.

In order to understand this structure, we need to analyze another feature of our map, the presence of a vanishing denominator.

\subsection{Focal points and lobes}

The two-dimensional map $T_{1}$ is defined in the whole plane except for the line $\delta_{S}: q_{2}=-q_{1}$ (and its preimages of any order), where the denominator in the first difference equation of system (9) vanishes. There is a particular point of the plane where both numerator and denominator of the first difference equation vanish and the map takes the form $0 / 0$. This point is the origin $Q(0,0)$ and it is called focal point. A focal point is defined as follows:

Definition 1. (Bischi et al. [31]). A point $Q=\left(x_{0}, y_{0}\right)$ is a focal point if at least one component of the map $T$ takes the form $0 / 0$ in $Q$ and there exist smooth simple arcs $\gamma(t)$, with $\gamma(0)=Q$, such that $\lim _{\tau \rightarrow 0} T(\gamma(\tau))$ is finite. The set of these finite values, obtained with different arcs $\gamma(t)$ through $Q$, is the prefocal set $\delta_{Q}$.

We have seen in the previous subsection how important it is to understand what happens in this point for explaining the basins structure in Figures 6 and 7. From Bischi et al. $[31,32,33]$ we know that to each focal point a prefocal curve $\delta_{Q}$ is associated, that is a set of points that are all mapped into the focal point by at least one of the inverses of the map. In our case the prefocal curve is a prefocal line and in particular it is the horizontal axis

$$
\delta_{Q}: q_{2}=0
$$

If the focal point is simple, then there is a one-to-one correspondence between the slope $m$ of an arc $\gamma$ through $Q$ and the point $\left(x^{\prime}, 0\right)$ in which its image crosses the prefocal line $\delta_{Q}$. One consequence, that is quite relevant to explain our basins configuration, is that if we consider an $\operatorname{arc}$ crossing $\delta_{Q}$ in two points, then there exists one rank-1 preimage forming a loop with a knot in $Q$, called lobe when this loop is the boundary of a basin of attraction. The two branches that issue from $Q$ have two different slopes, corresponding to the two points of the prefocal curve crossed by their images.

Our case is even more complicated than the one just described. In fact, our point is not simple (see the Appendix for details) and this implies that the correspondence between $\operatorname{arcs}$ through $Q$ and points of the prefocal line $\delta_{Q}$ is not one-to-one but two-to-one (see Bischi et al. [16, 33]). Then we have the following result.

Proposition 5. For any given point $\left(q_{1}, 0\right) \in \delta_{Q}$, the two slopes of the arcs through $Q$ are the following

$$
m_{ \pm}\left(q_{1}\right)=\frac{\alpha-2 q_{1} \pm \sqrt{\alpha^{2}-4 \alpha q_{1}}}{2 q_{1}} .
$$


The lobes through $Q$ are clearly visible in Figures 6 and 7. In the enlarged portion of the phase plane displayed in Figure 6, we can see that the number of these lobes is high and they seem to accumulate on the borders of the basins of attraction of feasible trajectories. This is a consequence of another feature of our map. In fact, our focal point $Q$ also belongs to the prefocal line $\delta_{Q}$ and so we have the following

Corollary 6. Any arc transverse to $\delta_{Q}$ has infinitely many preimages which are arcs through $Q$.

Now we are ready to explain the emergence of the complicated basins configuration of Figure 6.

\subsection{The emergence of lobes}

In this subsection we explain the emergence of lobes in our map. We focus on the gray lobes in Figure 6, that is the lobes corresponding to the basin of diverging trajectories $\beta(\infty)$.

We have that the $q_{2}$-axis is invariant (or a trapping set), in fact

$$
T_{1}\left(0, q_{2}\right)=\left(0, q_{2}-\frac{1}{2} c_{2} q_{2}^{2}\right)
$$

In particular, the $q_{2}$-axis (denoted by $\omega_{0}$ in Figure 8 , in accordance with the notation of Bischi et al. [16]) and its preimages of any rank separate the basin of diverging trajectories $B(\infty)$ from the basin of feasible trajectories $\beta(f)$. The lower curve side of the pseudo-triangle is one of the preimages of rank-1 of the $q_{2}$-axis (denoted by $\omega_{-1}$ ). This curve can be analytically computed, obtaining

$$
\omega_{-1}: q_{1}=h\left(q_{2}\right)=\sqrt{\frac{\alpha q_{2}}{\alpha c_{1}-1}}-q_{2},
$$

which represents a function in the $\left(q_{1}, q_{2}\right)$ plane.

The main gray lobe issuing from the focal point $Q$ is formed by the preimages of rank- 1 of the portion of $\beta(\infty)$ located between $\omega_{-1}$ and the prefocal line $\delta_{Q}$, namely those points not located in the region $Z_{0}$, that are dark gray in Figure 7.

If the portion of $\omega_{-1}$ delimiting the pseudo-triangle were located below the prefocal line, then we should not see any gray lobe inside the pseudo-triangle. This means that we can exactly calculate the value of the parameters characterizing the birth of the gray lobe (we recall that, thanks to Corollary 6 , once a lobe is born, other infinite lobes are also born with it). In fact we know the coordinates of the vertex $Q_{b}^{-1}$ and by considering the concavity plane we can state the following proposition

Proposition 7. The lobes corresponding to the basin of diverging trajectories $\beta(\infty)$ exist provided that $\alpha>\frac{1}{c_{1}}$ 


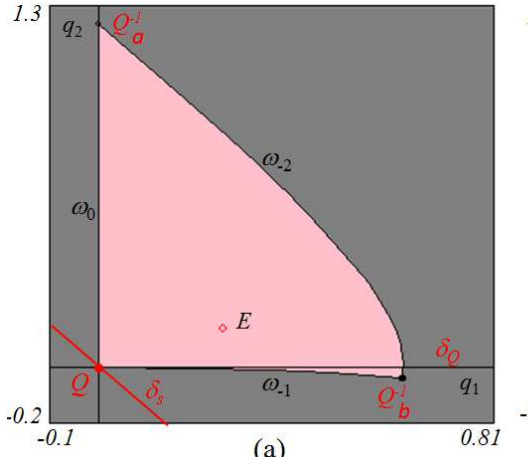

(a)

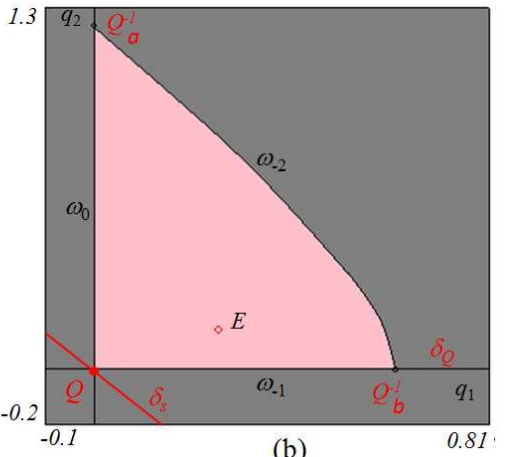

(b)

Figure 8: Left plot: for $\alpha=0$ the preimage $Q_{b}^{-1}$ of the focal point is below the $q_{1}$ axis, and there are no gray lobes in the basins configuration. Right plot: for $\alpha \approx \alpha_{g}$ the preimage $Q_{b}^{-1}$ of the focal point is located on the $q_{1}$ axis.

By applying this proposition to our example, we get the global bifurcation value of the speed of reaction

$$
\alpha_{g}=1 . \overline{1}
$$

where the vinculum (i.e. the horizontal bar over a decimal number) denotes a repeating group of digits (or a single digit as in our case).

The next set of figures gives a numerical confirmation of these results. In Figure $8 \mathrm{a}$, we used a value $\alpha=1$ and actually we can see that the preimage of the focal point $Q_{b}^{-1}$ is below the prefocal line and the portion of $\omega_{-1}$ delimiting the basin of feasible trajectories is below $\delta_{Q}$ too. As expected there are no gray lobes in the basins configuration (actually there are no lobes at all because the Nash Equilibrium is the only attractor).

In the next figure (Figure $8 \mathrm{~b}$ ) we used $\alpha \simeq \alpha_{g}$ and we can see that $Q_{b}^{-1}$ is located on the $q_{1}$-axis and the lower boundary of $\beta(f)$ is linear (a portion of the $q_{1}$-axis itself).

In Figure 9a, we used a value of the speed of adjustment slightly above the global bifurcation value $(\alpha=1.2) . Q_{b}^{-1}$ has now positive coordinates and the lower boundary of $B(f)$ is above the prefocal line. At first glance there are no significant differences in the basins configuration. Instead, by enlarging the region around the focal point, we can see a small gray lobe issuing from $Q$ (Figure $9 \mathrm{~b}$ ). This lobe is made up by preimages of rank- 1 of the portion of $B(\infty)$, which is above the prefocal line (and does not belong to region $Z_{0}$ ). Figure $9 \mathrm{c}$ further magnifies the region around $Q$. Moreover, from Corollary 6 we know that in our case a lobe cannot appear as a single one, as necessarily infinitely many lobes appear simultaneously. In Figure 9d, we can see one of these lobes located between the main lobe (denoted by $l_{M}$ ) and the $q_{2}$-axis. 

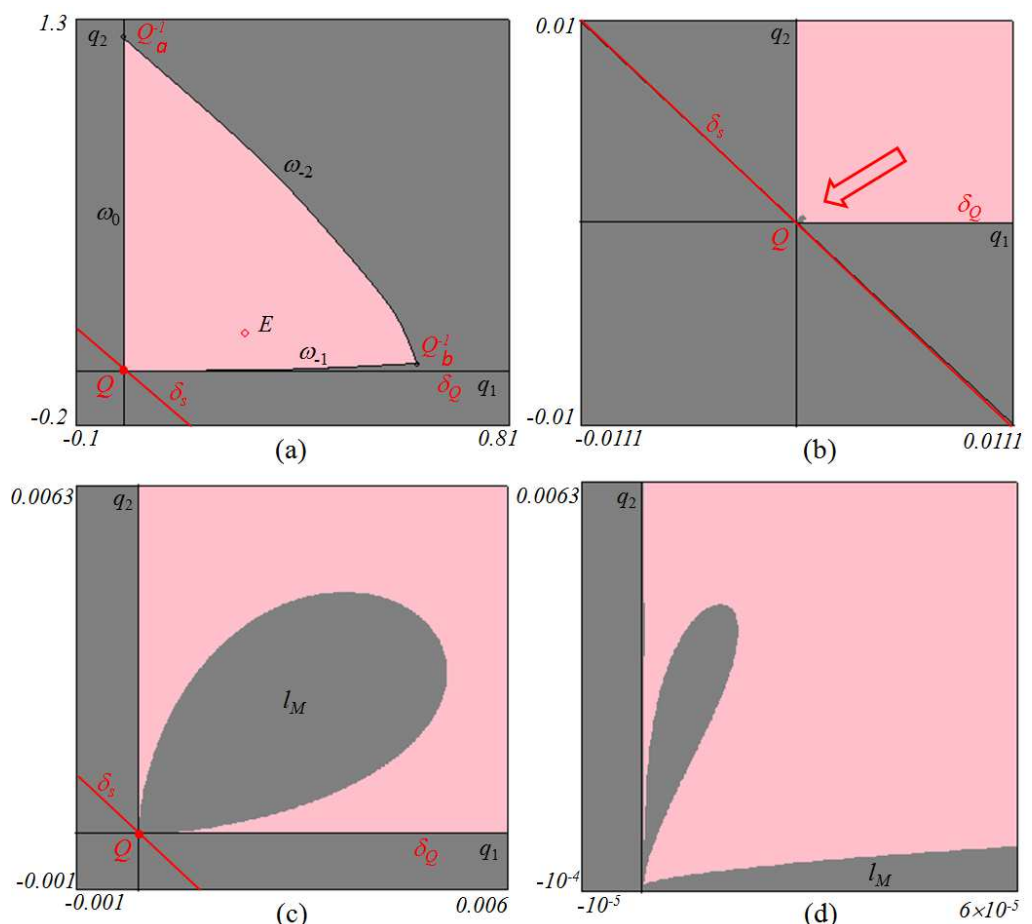

Figure 9: From top to bottom, left to right. (a) the value of the speed of adjustment slightly over the global bifurcation value $(\alpha=1.2)$. (b) Around the focal point there is a small gray lobe issuing from $Q$ (c) Further magnification of the region in (b) around $Q$. (d) Lobes located between the main lobe (denoted by $l_{M}$ ) and the $q_{2}$-axis. 


\section{Conclusions}

In this work, we introduced and studied a duopolistic market consisting of a firm following a gradient rule with endogenous reactivity and a firm following LMA. We showed that the steady state coincides with the Nash equilibrium and we provided conditions under which the equilibrium is stable. The duopoly we considered is comparable to that studied in [18], since in both cases one of the agents follows the gradient rule with endogenous reaction speed. However, in our contribution, the remaining player uses the LMA mechanism, while in [18] he computes the best response with static expectations, resulting more rational. This allowed us to compare the respective stability regions, and we found that, depending on the costs ratio, the stability region of the competition we proposed, involving the least rational players, can be larger than that of the duopoly studied in [18]. This scenario already emerged comparing different homogeneous oligopolies. Here we provided an example in which we have the same behavior for a heterogeneous competition. Moreover, we gave a further example for which two different roues toward chaotic dynamics, Neimark-Sacker and flip bifurcations, are possible. In the literature, this scenario seems to be connected to the presence of an agent adopting the gradient rule. In future works, we aim to study couplings of other decisional mechanisms, to investigate whether the appearance of the Neimark-Sacker bifurcation be necessarily linked to the presence of the gradient rule or not.

Finally, this particular combination of heuristics allowed us to provide a concrete example of a two dimensional dynamical system for which the theory developed by Bischi et. al about the maps with denominator can be applied and analytically studied. We have been able to investigate global properties such as multistability and appearance of lobes. The local analysis alone does not permit understanding the behavior of the trajectories, but through the analysis of the focal point, which in the present case is not simple, we provided analytical proof of the appearance of infinitely many lobes in the phase diagram.

In a future work we intend to study the same coupling of decisional rules but for an oligopoly consisting of more than 2 firms, considering both the situation in which the fractions of the firms that choose a specific rule is fixed and the situation in which each oligopolist can adapt over time the decisional rule to follow. Concerning this last goal, we remark that Droste et al. in [34] and Anufriev et al. in [26] proposed and studied a way to allow the firms to choose between different heuristics, which we aim to investigate in our future contribution. 


\section{Appendix}

Proof of Proposition 2. The proof is similar to that of Proposition 2 in [13]. To prove stability we introduce the Jacobian matrix of system (9)

$$
J\left(q_{1}, q_{2}\right)=\left(\begin{array}{cc}
1-\alpha c_{1}+\alpha q_{2}\left[\frac{q_{2}-q_{1}}{\left(q_{1}+q_{2}\right)^{3}}\right] & \alpha q_{1}\left[\frac{q_{1}-q_{2}}{\left(q_{1}+q_{2}\right)^{3}}\right] \\
\frac{1}{2}-c_{2}\left(q_{1}+q_{2}\right) & 1-c_{2}\left(q_{1}+q_{2}\right)
\end{array}\right),
$$

which, evaluated at (3), becomes

$$
J_{E}=J\left(q_{1}^{N}, q_{2}^{N}\right)=\left(\begin{array}{cc}
1-2 \alpha \frac{c_{1} c_{2}}{c_{1}+c_{2}} & \alpha c_{2}\left(\frac{c_{2}-c_{1}}{c_{1}+c_{2}}\right) \\
\frac{c_{1}}{c_{1}+c_{2}}-\frac{1}{2} & \frac{c_{1}}{c_{1}+c_{2}}
\end{array}\right) .
$$

The usual conditions for the local stability of the fixed point of a twodimensional discrete dynamical system are

$$
\left\{\begin{array}{l}
1-\operatorname{Tr}\left(J_{E}\right)+\operatorname{det}\left(J_{E}\right)>0 \\
1+\operatorname{Tr}\left(J_{E}\right)+\operatorname{det}\left(J_{E}\right)>0 \\
1-\operatorname{det}\left(J_{E}\right)>0
\end{array}\right.
$$

where

$$
\operatorname{Tr}\left(J_{E}\right)=\frac{2\left(1-\alpha c_{2}\right) c_{1}+c_{2}}{c_{1}+c_{2}}
$$

and

$$
\operatorname{det}\left(J_{E}\right)=\frac{\left(2-3 \alpha c_{2}\right) c_{1}+\alpha c_{2}^{2}}{2\left(c_{1}+c_{2}\right)},
$$

are respectively the trace and the determinant of matrix (18). After some algebraic manipulations, the first condition of (19) reduces to $c_{2} \alpha / 2>0$, which is indeed fulfilled for all positive $\alpha, c_{1}, c_{2}$. Likewise, the second and the third condition of (19) reduces to

$$
\frac{\alpha c_{2}\left(c_{2}-7 c_{1}\right)+8 c_{1}+4 c_{2}}{2\left(c_{1}+c_{2}\right)}>0
$$

and

$$
\frac{c_{2}\left(\alpha\left(3 c_{1}-c_{2}\right)+2\right)}{2\left(c_{1}+c_{2}\right)}>0
$$

To solve system (20), we divide the proof in two steps. First, we analyze the case in which either (20a) or (20b) is satisfied for all $\alpha$. Then we take into account the remaining cases. The final solution is obtained by the union of the two intermediate solutions.

We start noticing that denominators of (20a) and (20b) are both positive, so we need to check the positivity of numerators. When $0<c_{1} / c_{2} \leq 1 / 7$, all 
the terms of the numerator of (20a) are positive, while, for the numerator of (20b), condition $c_{2}\left(\alpha\left(3 c_{1}-c_{2}\right)+2\right)>0$ can be easily rewritten as $\alpha<\alpha_{n s}$.

Similarly, when $c_{1} / c_{2} \geq 1 / 3$ all the terms of the numerator of (20b) are positive, while, for the numerator of (20a), condition $\alpha c_{2}\left(c_{2}-7 c_{1}\right)+8 c_{1}+4 c_{2}>0$ straightforwardly gives $\alpha<\alpha_{f}$. Summarizing such conditions, we have that if

$$
\begin{aligned}
& 0<\frac{c_{1}}{c_{2}} \leq \frac{1}{7} \text { and } \alpha<\alpha_{n s}, \\
& \frac{c_{1}}{c_{2}} \geq \frac{1}{3} \text { and } \alpha<\alpha_{f},
\end{aligned}
$$

then conditions (20a) and (20b) are both satisfied. Now, we need to check positivity of system (20a)-(20b) for $c_{1} / c_{2} \in(1 / 7,1 / 3)$. In this case, we already proved that condition (20a) is equivalent to $\alpha<\alpha_{f}$ and that condition (20b) is equivalent to $\alpha<\alpha_{n s}$, so that system (20) requires $\alpha<\min \left\{\alpha_{n s}, \alpha_{f}\right\}$. To find $\min \left\{\alpha_{n s}, \alpha_{f}\right\}$, we check conditions for which $\alpha_{n s} \leq \alpha_{f}$, namely

$$
\begin{aligned}
\frac{2}{c_{2}-3 c_{1}}-\frac{8 c_{1}+4 c_{2}}{c_{2}\left(7 c_{1}-c_{2}\right)} & =\frac{2 c_{2}\left(7 c_{1}-c_{2}\right)-\left(8 c_{1}+4 c_{2}\right)\left(c_{2}-3 c_{1}\right)}{c_{2}\left(7 c_{1}-c_{2}\right)\left(c_{2}-3 c_{1}\right)} \\
& =\frac{6\left(c_{1}^{2}+3 c_{1} c_{2}-c_{2}^{2}\right)}{c_{2}\left(7 c_{1}-c_{2}\right)\left(c_{2}-3 c_{1}\right)} .
\end{aligned}
$$

Factorizing $c_{1}^{2}+3 c_{1} c_{2}-c_{2}^{2}$, we have

$$
\frac{6\left(c_{1}+c_{2}\right)\left(c_{1}-c_{2} / 4\right)}{4 c_{2}\left(c_{2}-3 c_{1}\right)\left(7 c_{1}-c_{2}\right)} \leq 0 .
$$

Since $c_{1} / c_{2} \in(1 / 7,1 / 3)$, the denominator of the l.h.s. of (22) is positive, so $(22)$ is satisfied for $c_{1} / c_{2} \leq 1 / 4$. This means that if $c_{1} / c_{2} \in(1 / 7,1 / 4]$, conditions (20) require $\alpha<\alpha_{n s}$, while if $c_{1} / c_{2} \in(1 / 4,1 / 3)$, conditions (20) require $\alpha<\alpha_{f}$. Combining (21) with such conditions we have (12).

Proof of Proposition 3. Let us suppose that $c_{1} / c_{2} \leq 1 / 4$. Model (9) is stable for $\alpha<\alpha_{n s}$ while model (13) is stable for $\alpha<\alpha_{n s}^{T}$. We have that

$$
\alpha_{n s}^{T}-\alpha_{n s}=\frac{2\left(c_{1}+c_{2}\right)}{\left(c_{2}-c_{1}\right)^{2}}-\frac{2}{c_{2}-3 c_{1}}=\frac{8 c_{1}^{2}}{\left(c_{2}-c_{1}\right)^{2}\left(3 c_{1}-c_{2}\right)}
$$

is negative since $\left(3 c_{1}-c_{2}\right)<0$ for $c_{1} / c_{2}<1 / 4$. This says that $\alpha_{n s}^{T}<\alpha_{n s}$ and then the stability region of (9) is larger than that of model (13) when $c_{1} / c_{2} \leq 1 / 4$.

Let us consider $1 / 4<c_{1} / c_{2}<1 / 3$. Model (9) is stable for $\alpha<\alpha_{f}$ while model (13) is again stable for $\alpha<\alpha_{n s}^{T}$. We have that

$$
\alpha_{n s}^{T}-\alpha_{f}=\frac{2\left(c_{1}+c_{2}\right)}{\left(c_{2}-c_{1}\right)^{2}}-\frac{8 c_{1}+4 c_{2}}{c_{2}\left(7 c_{1}-c_{2}\right)}=\frac{-8 c_{1}^{3}+26 c_{1}^{2} c_{2}+12 c_{1} c_{2}^{2}-6 c_{2}^{3}}{c_{2}\left(c_{2}-c_{1}\right)^{2}\left(7 c_{1}-c_{2}\right)}
$$

where the denominator is positive for $1 / 4<c_{1} / c_{2}<1 / 3$, while

$$
-8 c_{1}^{3}+26 c_{1}^{2} c_{2}+12 c_{1} c_{2}^{2}-6 c_{2}^{3}=-8 c_{2}^{3}\left(c_{1} / c_{2}-s_{0}\right)\left(c_{1} / c_{2}-s_{1}\right)\left(c_{1} / c_{2}-s_{2}\right)
$$


where

$$
s_{0}<0<s_{1}<1 / 4<1 / 3<3<s_{2} .
$$

Then (24) is negative for $1 / 4<c_{1} / c_{2}<s_{1} \approx 0.3108$ and positive for $s_{1}<$ $c_{1} / c_{2}<1 / 3$, so that (23) is negative for $1 / 4<c_{1} / c_{2}<s_{1} \approx$ and positive for $s_{1}<c_{1} / c_{2}<1 / 3$, which allows concluding that the stability region of (9) is larger than that of model (13) when $0<c_{1} / c_{2} \leq s_{1}$.

When $1 / 3<c_{1} / c_{2}<3$, model (9) is stable for $\alpha<\alpha_{f}$ while model (13) is stable for $\alpha<\alpha_{f}^{T}$. We have that

$$
\alpha_{f}^{T}-\alpha_{f}=\frac{4\left(c_{1}+c_{2}\right)}{4 c_{1} c_{2}-\left(c_{2}-c_{1}\right)^{2}}-\frac{8 c_{1}+4 c_{2}}{c_{2}\left(7 c_{1}-c_{2}\right)}=-\frac{8 c_{1}\left(c_{2}-c_{1}\right)^{2}}{c_{2}\left(7 c_{1}-c_{2}\right)\left(c_{1}^{2}-6 c_{1} c_{2}+c_{2}^{2}\right)}
$$

which is positive since the numerator is positive, $-c_{2}\left(7 c_{1}-c_{2}\right)<0$ for $1 / 3<$ $c_{1} / c_{2}<3$ and $c_{1}^{2}-6 c_{1} c_{2}+c_{2}^{2}<0$ for

$$
3-2 \sqrt{2}<\frac{1}{3}<\frac{c_{1}}{c_{2}}<3<3+2 \sqrt{2} .
$$

This implies that $\alpha_{f}^{T} \geq \alpha_{f}$ and the stability region of (9) is smaller than that of $(13)$.

The last situation concerns $c_{1} / c_{2}>3$, in which model (9) is stable for $\alpha<\alpha_{f}$ while model (13) is stable for $\alpha<\alpha_{n s}^{T}$. We have that (23) is positive provided that $3<c_{1} / c_{2}<s_{2} \approx 3.6081$, since both its denominator and (24) are positive. This allows concluding that $\alpha_{n s}^{T} \geq \alpha_{f}$ and the stability region of (9) is smaller than that of (13) for $3<c_{1} / c_{2}<s_{2}$. Conversely, we have that (23) is negative when $c_{1} / c_{2}>s_{2}$, and this implies that the stability region of (9) is again larger than that of (13). Combining this last result with the previous one, can we conclude.

Proof of Proposition 4. In order to calculate the number of preimages of points belonging to the $q_{2}$-axis of the phase plane, we consider a generic point $\left(0, \bar{q}_{2}\right)$. We use these two coordinates in system (9) to replace $q_{1}(t+1)$ and $q_{2}(t+1)$, respectively. We obtain the system

$$
\left\{\begin{array}{l}
0=q_{1}+\alpha q_{1}\left[\frac{q_{2}}{\left(q_{1}+q_{2}\right)^{2}}-c_{1}\right] \\
\bar{q}_{2}=\frac{1}{2} q_{2}+\frac{1}{2}\left[1-c_{2}\left(q_{1}+q_{2}\right)\right]\left(q_{1}+q_{2}\right)
\end{array}\right.
$$

and we must find the coordinates $\left(q_{1}, q_{2}\right)$ of the points that solve it. We can rearrange the first equation as follows

$$
q_{1}\left[1-\alpha c_{1}+\frac{\alpha q_{2}}{\left(q_{1}+q_{2}\right)^{2}}\right]=0 .
$$

Equation (26) is a product that becomes zero when at least one of the factors is zero. So, we must consider two cases. 
- Case $q_{1}=0$

By substituting $q_{1}=0$ in the second equation of the system (25), after some algebraic calculations we obtain the following second degree equation in $q_{2}$

$$
\frac{1}{2} c_{2} q_{2}^{2}-q_{2}+\bar{q}_{2}=0
$$

whose roots are

$$
q_{2}^{*}=\frac{1 \pm \sqrt{1-2 c_{2} \bar{q}_{2}}}{c_{2}} .
$$

These roots are real provided that

$$
\bar{q}_{2} \leq \bar{y}_{1}
$$

where $\bar{y}_{1}=1 / 2 c_{2}$. In such a case, the two preimages of the point $\left(0, \bar{q}_{2}\right)$ are given by

$$
\left(0, \frac{1+\sqrt{1-2 c_{2} \bar{q}_{2}}}{c_{2}}\right) \text { and }\left(0, \frac{1-\sqrt{1-2 c_{2} \bar{q}_{2}}}{c_{2}}\right) \text {. }
$$

- Case $1-\alpha c_{1}+\frac{\alpha q_{2}}{\left(q_{1}+q_{2}\right)^{2}}=0$

The condition characterizing this case cannot be immediately used in the second equation of system (25). We can rearrange this condition as follows

$$
\left(q_{1}+q_{2}\right)^{2}=\frac{\alpha q_{2}}{\alpha c_{1}-1} .
$$

Since the l.h.s. of (28) is positive, the r.h.s. has to be positive as well, and this requires

$$
\alpha c_{1} \geq 1 .
$$

If (29) is false, then we can conclude the third part of the thesis.

Similarly with the second equation of system (25) we can write

$$
\left(q_{1}+q_{2}\right)^{2}=\frac{2 q_{2}+q_{1}-2 \bar{q}_{2}}{c_{2}} .
$$

By combining the right sides of equations (28) and (30) and by solving the consequent equation for $q_{1}$ we get

$$
q_{1}=\frac{\alpha c_{2} q_{2}}{\alpha c_{1}-1}-2 q_{2}+2 \bar{q}_{2},
$$

under condition (29). We can use it in the second equation of system (25) obtaining a second degree equation in $q_{2}$

$$
a q_{2}^{2}+b q_{2}+c=0
$$


with

$$
\begin{aligned}
& a=-\frac{\alpha^{2} c_{2}^{3}}{2\left(\alpha c_{1}-1\right)^{2}}-\frac{c_{2}}{2}+\frac{\alpha c_{2}^{2}}{\alpha c_{1}-1}, \\
& b=\frac{\alpha c_{2}}{2\left(\alpha c_{1}-1\right)}+2 c_{2} \bar{q}_{2}-2 \frac{\alpha c_{2}^{2} \bar{q}_{2}}{\alpha c_{1}-1}, \\
& c=-2 c_{2} \bar{q}_{2}^{2} .
\end{aligned}
$$

The discriminant of equation (31) is

$$
\Delta=\frac{\alpha^{2} c_{2}^{2}}{4\left(\alpha c_{1}-1\right)^{2}}+2 \frac{\alpha c_{2}^{2} \bar{q}_{2}}{\alpha c_{1}-1}-2 \frac{\alpha^{2} c_{2}^{3} \bar{q}_{2}}{\left(\alpha c_{1}-1\right)^{2}}
$$

which is positive if $\alpha c_{2}^{2}\left(\alpha-8 \bar{q}_{2}+8 \alpha c_{1} \bar{q}_{2}-8 \alpha c_{2} \bar{q}_{2}\right) \geq 0$. The last inequality is satisfied for all the $\bar{q}_{2} \geq 0$ when $\alpha\left(c_{1}-c_{2}\right) \geq 1$, and for $\bar{q}_{2} \leq \bar{y}_{2}$ when $\alpha\left(c_{1}-c_{2}\right)<1$. We recall that each of these last inequalities have to be considered under condition (29).

To evaluate the total number of preimages, we notice that when $\alpha\left(c_{1}-c_{2}\right)>$ 1 is true, then automatically $\alpha c_{1}>1$, so condition (29) is satisfied. Then, for $\alpha c_{1}>1+\alpha c_{2}$ we always have at least two preimages, and we have four preimages when (27) is satisfied.

Conversely, when $1<\alpha c_{1}<1+\alpha c_{2}$ both $\bar{y}_{1}<\bar{y}_{2}$ and $\bar{y}_{2}<\bar{y}_{1}$ are possible. In particular, we have $\bar{y}_{1}<\bar{y}_{2}$ if $1+3 / 4 \alpha c_{1}<\alpha c_{1}<\alpha c_{2}$. Conversely, we have $\bar{y}_{2}<\bar{y}_{1}$ if $1<\alpha c_{1}<1+3 / 4 \alpha c_{2}$. Recalling that in this case the discriminant of (31) is positive provided that $\bar{q}_{2}<\bar{y}_{2}$ and recalling (27), we can obtain the second part of the thesis.

Proof of Proposition 5. Our map (9) belongs to the family of maps $\left(x^{\prime}, y^{\prime}\right)=$ $(N(x, y) / D(x, y), G(x, y))$, called maps with denominator in Bischi et al. [31, 32, 33]. These maps are defined in the whole phase plane except for the points in which $D(x, y)=0$ and their preimages of any rank. These points form a set called set of non-definition $\delta_{S}$ and in our a case it is the line of equation

$$
\delta_{S}: q_{2}=-q_{1} .
$$

If a point $Q=\left(x_{0}, y_{0}\right)$ belonging to the set of non-definition is such that $N\left(x_{0}, y_{0}\right)=0$ then we are in presence of a focal point (see Definition 1). It is immediate to see that our map $T_{1}$ owns one focal point $Q$ given by

$$
Q=(0,0)
$$

and its prefocal set can be obtained by substituting the coordinates of the focal point into the first equation of the system (9), obtaining

$$
\delta_{Q}: q_{2}=0 .
$$

A focal point $Q$ is simple provided that $\bar{N}_{y} \bar{D}_{x}-\bar{N}_{x} \bar{D}_{y} \neq 0$, where the subscripts indicate partial derivatives calculated in $Q$. In this case there exists a one-toone correspondence between the slope $m$ of an $\operatorname{arc} \gamma$ through $Q$ and the point 
$(x, G(Q))$ in which its image crosses $\delta_{Q}$

$$
m \rightarrow(x(m), G(Q)), \quad \text { with } \quad x(m)=\frac{\bar{N}_{y}+m \bar{N}_{x}}{\bar{D}_{y}+m \bar{D}_{x}} .
$$

This result can be proved if we consider a simple arc $\gamma$ transverse to $\delta_{S}$, that we can write as follows:

$$
\gamma(\tau):\left\{\begin{array}{l}
x(\tau)=x_{0}+\xi_{1} \tau+\xi_{2} \tau^{2}+\ldots \\
y(\tau)=y_{0}+\eta_{1} \tau+\eta_{2} \tau^{2}+\ldots
\end{array} \quad \tau \neq 0 .\right.
$$

If $N(x, y)$ and $D(x, y)$ are both smooth functions, so that

$$
\begin{aligned}
& N(x, y)=\bar{N}_{x}\left(x-x_{0}\right)+\bar{N}_{y}\left(y-y_{0}\right)+O_{2} \\
& D(x, y)=\bar{D}_{x}\left(x-x_{0}\right)+\bar{D}_{y}\left(y-y_{0}\right)+O_{2}^{\prime}
\end{aligned}
$$

with $\mathrm{O}_{2}$ and $\mathrm{O}_{2}^{\prime}$ terms of higher order, and the arc crosses through the simple focal point $Q$ we get

$$
\lim _{\tau \rightarrow 0} \frac{N(\gamma(\tau))}{D(\gamma(\tau))}=\frac{\eta_{1} \bar{N}_{y}+\xi_{1} \bar{N}_{x}}{\eta_{1} \bar{D}_{y}+\xi_{1} \bar{D}_{x}}
$$

from which (32) follows if we define $m=\xi_{1} / \eta_{1}$.

The focal point of our map is not simple. In fact we have that all the partial derivatives $\left(\bar{N}_{q_{1}}, \bar{N}_{q_{2}}, \bar{D}_{q_{1}}, \bar{D}_{q_{2}}\right)$ vanish when they are calculated at the focal point.

As shown in Bischi et al. [33], in such a case the correspondence between the slopes of $\operatorname{arcs}$ through $Q$ and the points of $\delta_{Q}$ is two-to-one. So we need the second order terms in the expansions of $N$ and $D$ for deriving the $x$-coordinate of the point $(x, G(Q))$ in which the image of the $\operatorname{arc}$ crosses $\delta_{Q}$, obtaining

$$
m \rightarrow(x(m), G(Q)), \quad \text { with } \quad x(m)=\frac{\bar{N}_{y y}+2 m \bar{N}_{x y}+\bar{N}_{x x} m^{2}}{\bar{D}_{y y}+2 m \bar{D}_{x y}+\bar{D}_{x x} m^{2}} .
$$

From (33) we can get the two values of the slopes

$$
m_{ \pm}(x)=\frac{-\left(\bar{N}_{x y}-x \bar{D}_{x y}\right) \pm \sqrt{\Delta}}{\left(\bar{N}_{x x}-x \bar{D}_{x x}\right)}
$$

that exist when $\Delta=\left[\left(\bar{N}_{x y}-x \bar{D}_{x y}\right)^{2}-\left(\bar{N}_{x x}-x \bar{D}_{x x}\right)\left(\bar{N}_{y y}-x \bar{D}_{y y}\right)\right]>0$. The second derivatives of our map (6) are the following

$$
\begin{array}{lll}
\bar{N}_{q_{1} q_{1}}=0 & \bar{N}_{q_{1} q_{2}}=\alpha & \bar{N}_{q_{2} q_{2}}=0 \\
\bar{D}_{q_{1} q_{1}}=2 & \bar{D}_{q_{1} q_{2}}=2 & \bar{D}_{q_{2} q_{2}}=2
\end{array}
$$

that permit us to obtain the two slopes

$$
m_{ \pm}\left(q_{1}\right)=\frac{\alpha-2 q_{1} \pm \sqrt{\alpha^{2}-4 \alpha q_{1}}}{2 q_{1}} .
$$


Proof of Corollary 6. The proof comes from Bischi et al. (2001), in which it is noted that any arc through $Q$ and transverse to $\delta_{Q}$ like all other arcs crossing $\delta_{Q}$ has two rank-1 preimages again crossing $\delta_{Q}$ in $Q$. Any arc through $Q$, according to the relation (17), has two preimages through $Q$ again. We can go on iteratively ad infinitum.

Proof of Proposition \%. The proof is a direct consequence of the fact that $\omega_{-1}$ is a continuous function in the $\left(q_{2}, q_{1}\right)$ plane. Then, if the vertex $Q_{b}^{-1}$ of the pseudo-triangle is above the prefocal line $\delta_{Q}$, the whole side is above it (and the opposite if the vertex is below it). So the gray lobes appear when the $q_{2}$ coordinate of $Q_{b}^{-1}$ is equal to 0 , that is when $\alpha=1 / c_{1}$ and the portion of $\omega_{-1}$ delimiting the pseudo-triangle is above the prefocal line when $\alpha>\frac{1}{c_{1}}$, q.e.d.

\section{Bibliography}

[1] Cournot AA. Researches into the principles of the theory of wealth. Engl. Trans., Irwin Paper Back Classics in Economics; 1963.

[2] Rand D. Exotic phenomena in games and duopoly models. J Math Econom 1978;5(2):173-84.

[3] Poston T, Stewart I. Catastrophe theory and its applications. London: Pitman Ltd.; 1978.

[4] Puu T. Chaos in duopoly pricing. Chaos, Solitons Fractals 1991;1(6):573 -81 .

[5] Silvestre J. A model of general equilibrium with monopolistic behavior. J Econ Theory 1977;16:425-42.

[6] Tuinstra J. A price adjustment process in a model of monopolistic competition. Int Game Theory Rev 2004;6(3):417-42.

[7] Bischi GI, Naimzada A, Sbragia L. Oligopoly games with local monopolistic approximation. J Econ Behav Organ 2007;62(3):371-88.

[8] Naimzada A, Sbragia L. Oligopoly games with nonlinear demand and cost functions: two boundedly rational adjustment processes. Chaos, Solitons Fractals 2006;29(3):707 -22.

[9] Naimzada A, Ricchiuti G. Monopoly with local knowledge of demand function. Econ Model 2011;28(1-2):299-307.

[10] Varian HR. Microeconomic Analysis. W. W. Norton; 1992.

[11] Corchon LC, Mas-Colell A. On the stability of best reply and gradient systems with applications to imperfectly competitive models. Econ Let $1996 ; 51(1): 59-65$. 
[12] Angelini N, Dieci R, Nardini F. Bifurcation analysis of a dynamic duopoly model with heterogeneous costs and behavioural rules. Math Comput Simul 2009;79(10):3179-96.

[13] Cavalli F, Naimzada A. A cournot duopoly game with heterogeneous players: Nonlinear dynamics of the gradient rule versus local monopolistic approach. Appl Math Comput 2014; doi: http://dx.doi.org/10.1016/j.amc.2014.10.031.

[14] Bischi GI, Naimzada A. Global analysis of a dynamic duopoly game with bounded rationality. In: Filar J, Gaitsgory V, Mizukami K, editors. Adv. in Dyn. Games and Appl. Shonan Village Ctr; 2000, p. 361-85. 7th Int. Symp. on Dyn. Games and Appl.

[15] Bischi GI, Gallegati M, Naimzada A. Symmetry-breaking bifurcations and representative firm in dynamic duopoly games. Ann Oper Res 1999;89:25372 .

[16] Bischi GI, Kopel M, Naimzada A. On a rent-seeking game described by a non-invertible iterated map with denominator. Nonlinear Anal 2001;47(8):5309-24.

[17] Agiza HN, Hegazi AS, Elsadany AA. Complex dynamics and synchronization of a duopoly game with bounded rationality. Math Comput Simul 2002;58(2):133-46.

[18] Tramontana F. Heterogeneous duopoly with isoelastic demand function. Econ Model 2010;27(1):350-7.

[19] Askar SS. Complex dynamic properties of cournot duopoly games with convex and log-concave demand function. Oper Res Lett 2014;42(1):85-90.

[20] Askar SS. The rise of complex phenomena in cournot duopoly games due to demand functions without inflection points. Commun Nonlinear Sci Numer Simul 2014;19(6):1918-25.

[21] Leonard D, Nishimura K. Nonlinear dynamics in the cournot model without full information. Ann Oper Res 1999;89:165-73.

[22] Den-Haan WJ. The importance of the number of different agents in a heterogeneous asset-pricing model. J Econ Dyn Control 2001;25(5):72146.

[23] Agiza HN, Elsadany AA. Nonlinear dynamics in the cournot duopoly game with heterogeneous players. Physica A 2003;320:512-24.

[24] Agiza HN, Elsadany AA. Chaotic dynamics in nonlinear duopoly game with heterogeneous players. Appl Math Comput 2004;149(3):843-60. 
[25] Dubiel-Teleszynski T. Nonlinear dynamics in a heterogeneous duopoly game with adjusting players and diseconomies of scale. Comm Nonlinear Sci and Numer Simul 2011;16(1):296-308.

[26] Anufriev M, Kopanyi D, Tuinstra J. Learning cycles in bertrand competition with differentiated commodities and competing learning rules. J Econ Dyn Control 2013;37(12):2562-81.

[27] Cavalli F, Naimzada A. Nonlinear dynamics and speed of convergence of an heterogeneous cournot duopoly with a local monopolistic approach versus a best-response approach with different degrees of rationality; 2014. In preparation.

[28] Naimzada A, Tramontana F. Controlling chaos through local knowledge. Chaos, Solitons Fractals 2009;42(4):2439 -49.

[29] Mira C, Gardini L, Barugola A, Cathala J. Chaotic Dynamics in TwoDimensional Noninvertible Maps. World Scientific; 1996.

[30] Abraham R, Gardini L, Mira C. Chaos in Discrete Dynamical Systems (a visual introduction in two Dimensions). Springer; 1996.

[31] Bischi G, Gardini L, Mira C. Plane maps with denominator. i. some generic properties. Int J Bifurc Chaos Appl Sci Eng 1999;9(1):119-53.

[32] Bischi G, Gardini L, Mira C. Plane maps with denominator. part ii: Noninvertible maps with simple focal points. Int J Bifurc Chaos Appl Sci Eng $2003 ; 13(8): 2553-277$.

[33] Bischi G, Gardini L, Mira C. Plane maps with denominator. part iii: Nonsimple focal points and related bifurcations.qe. Int J Bifurc Chaos Appl Sci Eng 2005;15(2):451-96.

[34] Droste E, Hommes C, Tuinstra J. Endogenous fluctuations under evolutionary pressure in cournot competition. Games and Economic Behavior 2002;40:232-69. 